Cite this: RSC Adv., 2017, 7, 5764

Received 16th November 2016 Accepted 5th January 2017

DOI: 10.1039/c6ra26879j

www.rsc.org/advances

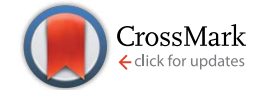

\section{A sensitive, uniform, reproducible and stable SERS substrate has been presented based on $\mathrm{MoS}_{2} \mathrm{CAg}$ nanoparticles@pyramidal silicon}

\author{
Shouzhen Jiang, $\dagger^{* a}$ Jia Guo, $\dagger^{\mathrm{a}}$ Chao Zhang, ${ }^{a}$ Chonghui $\mathrm{Li}^{\mathrm{a}}$ Minghong Wang, ${ }^{\mathrm{a}}$ \\ Zhen Li, ${ }^{a}$ Saisai Gao, ${ }^{a}$ Peixi Chen, ${ }^{a}$ Haipeng Si ${ }^{b}$ and Shicai $\mathrm{Xu}^{\mathrm{c}}$
}

\begin{abstract}
By combining the excellent surface-enhanced Raman scattering (SERS) activity of Ag nanoparticles (AgNPs), the well-separated pyramid arrays of the pyramidal silicon (PSi) and unique physical/chemical properties of molybdenum disulfide $\left(\mathrm{MoS}_{2}\right)$, the $\mathrm{MoS}_{2}$ @AgNPs@PSi substrate shows high performance in terms of sensitivity, uniformity, reproducibility and stability. By using rhodamine 6G (R6G) as probe molecule, the SERS results indicate that the MoS $_{2} @ A g N P s @ P S i$ substrate is superior to the AgNPs@PSi, AgA@PSi (the second annealing of the AgNPs@PSi) and the $\mathrm{MoS}_{2} @ A g N P s @$ flat-Si substrate. The MoS 2 AANPs@PSi substrate also shows the reasonable linear response between the Raman intensity and R6G concentration. The maximum deviations of SERS intensities from 20 positions on a same $\mathrm{MoS}_{2} @$ AgNPs@PSi substrate and $10 \mathrm{MoS}_{2}$ @AgNPs@PSi substrates in different batches are less than 7.6\% and $9 \%$, respectively, revealing the excellent uniformity and reproducibility of the substrate. Besides, the SERS substrate has a good stability, the Raman intensity of the MoS 2 @AgNPs@PSi substrate only drop by $15 \%$ in a month. The corresponding experimental and theoretical results suggest that our proposed MoS $_{2}$ @AgNPs@PSi substrate is expected to offer a new and practical way to accelerate the development of label-free SERS detection.
\end{abstract}

\section{Introduction}

In recent decades, surface-enhanced Raman scattering (SERS) as a very important analytical technique for molecular detection has received increasing attention. ${ }^{1-3}$ About SERS, it is now well accepted that the plasmonic coupling effect at the nanometre gap junction between metal particles induces enormous electromagnetic enhancement that allows SERS signal to be detected with high sensitivity. ${ }^{4-8}$ However, the non homogeneous structure and instability of SERS substrate based on metal nanoparticles restricts the practical application and commercialization of SERS technology. ${ }^{9}$ An ideal SERS nanostructure for practical applications should induce a high signal enhancement, generate a uniform and reproducible response, and have a long-term stability. ${ }^{\mathbf{1 0}}$

Layered transition metal dichalcogenides (LTMDs) have received much attention due to their remarkable optoelectronic properties. ${ }^{11-13}$ Molybdenum disulfide $\left(\mathrm{MoS}_{2}\right)$ belongs to the

\footnotetext{
${ }^{a}$ School of Physics and Electronics, Shandong Normal University, Jinan 250014, China. E-mail: jiang_sz@126.com

${ }^{b}$ Department of Orthopaedics, Qilu Hospital, Shandong University, 107 Wenhuaxilu Street, Jinan 250012, China

${ }^{c}$ Shandong Provincial Key Laboratory of Biophysics, College of Physics and Electronic Information, Dezhou University, Dezhou 253023, PR China

$\dagger$ Both authors contributed equally to this work.
}

family of LTMDs. The layered $\mathrm{MoS}_{2}$ has unique physical, optical and electrical properties, making it very interesting for applying as a promising supporting material to stabilize metal nanoparticles, forming hierarchical composites. ${ }^{\mathbf{1 4 - 1 7}}$ The composites $\left(\mathrm{MoS}_{2} /\right.$ metal nanoparticles) sometimes offer synergistic effects to the intrinsic properties of the metal nanoparticles, making the composites much more attractive in applications of surfaceenhanced Raman scattering (SERS) than the metal nanoparticles alone. ${ }^{18,19}$ Compared with graphene, $\mathrm{MoS}_{2}$ can be get in a low temperature that can effectively reduce energy consumption and makes it easily combine with a traditional metallic substrate, ${ }^{\mathbf{2 0 - 2 4}}$ which allows us to take advantage of both electromagnetic mechanism (EM) and a chemical mechanism (CM). In our work, we directly grew $\mathrm{MoS}_{2}$ on $\mathrm{Ag}$ nanoparticles (AgNPs) by thermally decomposing the precursor of $\left(\mathrm{NH}_{4}\right)_{2} \mathrm{MoS}_{4}$. The $\mathrm{MoS}_{2}$ films exhibits three advantages in our work: first, the $\mathrm{MoS}_{2}$ films can provide the chemical enhancement and efficient adsorption for various target molecules, so the sensitivity of the substrate is enhanced; second, the $\mathrm{MoS}_{2}$ layer reduces the additional signal by isolating the metal nanoparticles from the R6G molecules. This characteristic of $\mathrm{MoS}_{2}$ will enhance the uniformity of SERS signals; third, the $\mathrm{MoS}_{2}$ films can isolate AgNPs from surrounding environment, effectively protect AgNPs from oxidation and endow the hybrid system a good stability and long lifetime. Thus a sensitive, uniform and stable SERS substrate is to be formed. 
In order to further enhance the sensitivity of the detection of Raman signals, porous Si has been intensively chosen to serve as SERS active substrate in recent years. ${ }^{25-29}$ It has been demonstrated that porous Si possessing large specific area and governable nanoporous structure can effective increase the number of hot spots and further enhance the sensitivity of the SERS signals. So all kinds of methods including laser interference lithography, ${ }^{30}$ chemical method, ${ }^{31}$ metal-assisted chemical etching, ${ }^{32}$ reactive ion etching ${ }^{33}$ have been carried out to fabricate the porous Si. The method of laser interference lithography or reactive ion etching can produce well-defined Si nanopattern. However these methods are time-consuming and expensive. Uniform porous Si with small structure can be produced with the chemical method or metal-assisted chemical etching. Although these methods relatively lost-cost, but they require multiple steps and multifarious strong acids in the preparation process. In our work, a relatively simple and lost-cost method was used to obtain the pyramidal Si (PSi) SERS substrate. PSi possessing well-separated pyramid arrays different from the traditional pillar arrays. The scattering area of PSi is very large and the incident laser is able to produce an effective oscillation among the valleys of PSi, which can further enhance the sensitivity of SERS substrate. ${ }^{34,35}$ Besides, the PSi possessing well-separated pyramid arrays, which can make contribution to the uniformity of the substrate.

Herein, we combine $\mathrm{MoS}_{2}$, AgNPs and PSi forming the $\mathrm{MoS}_{2} @ A g N P s @ P S i$ substrate. By using rhodamine 6G (R6G) as a probe molecule, we experimentally realize and prove the high sensitivity, excellent uniformity and reproducibility and longterm stability of the $\mathrm{MoS}_{2} @ A g N P s @ P S i$ substrate.

\section{Experimental}

\subsection{The preparation of the $\mathrm{MoS}_{2} @ A g N P s @ P S i$ substrate}

The preparation processes of the $\mathrm{MoS}_{2} @ A g N P s @ P S i$ substrate are summarized in Fig. 1. We fabricate PSi substrates through wet texturing boron-doped single crystal silicon wafer with the assist of the $\mathrm{NaOH}$ solution. Subsequently, all the PSi substrates were cleaned by acetone, alcohol and deionized water in ultrasonic cleaner for $30 \mathrm{~min}$, respectively. By thermal-evaporation system, continuous Ag films was deposited on PSi substrate. In order to obtain appropriate AgNPs, the PSi with Ag films was placed in the quartz tube and the pressure was pumped to $10^{-3}$ $\mathrm{Pa}$. Then $40 \mathrm{sccm}$ Ar was introduced into the quartz tube and the temperature has been raised to $500{ }^{\circ} \mathrm{C}$ for annealing $30 \mathrm{~min}$. Finally, the temperature of the quartz tube was fast cooled down to room temperature by opening the lid of furnace. Through these steps, the AgNPs@PSi substrate was obtained. The $10 \mathrm{~mL}$ dimethylformamide was added into the high purity of $\left(\mathrm{NH}_{4}\right)_{2} \mathrm{MoS}_{4}$ (purity of $99.99 \% ; 1 \mathrm{~g}$ ) powder to form a $1 \mathrm{wt} \%$ solution. Next, the $\left(\mathrm{NH}_{4}\right)_{2} \mathrm{MoS}_{4}$ solution was treated by sonication in ultrasonic cleaner for $20 \mathrm{~min}$ with the power of $80 \mathrm{~W}$ to break down the undissolved particles. The $\left(\mathrm{NH}_{4}\right)_{2} \mathrm{MoS}_{4}$ solution was immediately deposited on the AgNPs@PSi substrate by using a spin-coating method. In order to get the $\mathrm{MoS}_{2}$ @AgNPs@PSi substrate, the thermally decomposing process was performed through the second annealing. First, the $\left(\mathrm{NH}_{4}\right)_{2} \mathrm{MoS}_{4}$

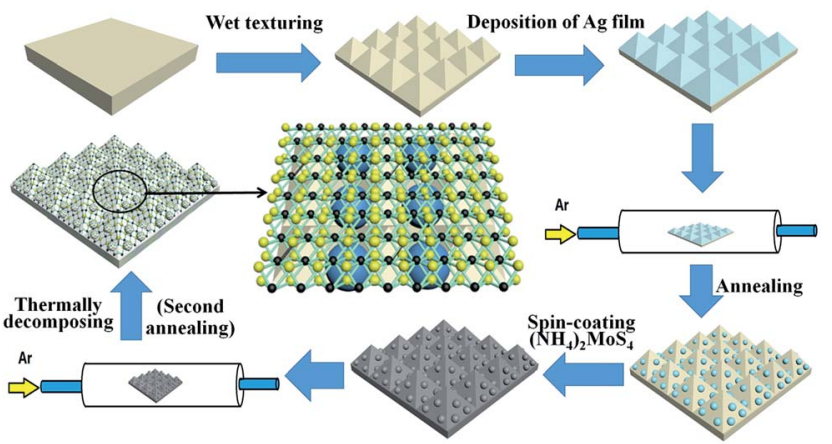

Fig. 1 The preparation processes of the $\mathrm{MoS}_{2} @$ AgNPs@PSi substrate.

membrane was placed in the quartz tube and the pressure was pumped to $10^{-3} \mathrm{~Pa}$. Second, $80 \mathrm{sccm}$ Ar was introduced into the tube and the temperature reached $500{ }^{\circ} \mathrm{C}$ for annealing $90 \mathrm{~min}$. Third, the tube was fast cooled down to room temperature by opening the furnace. In order to demonstrate the effect of the $\mathrm{MoS}_{2}$ layer, we also prepared AgA@PSi (the second annealing of the AgNPs@PSi). The AgNPs@PSi substrate without coating $\left(\mathrm{NH}_{4}\right)_{2} \mathrm{MoS}_{4}$ solution was directly placed in the quartz tube for the second annealing. The AgA@PSi substrate was fabricated with the same method as preparing the $\mathrm{MoS}_{2} @ A g N P s @ P S i$ substrate. Besides, we prepared the $\mathrm{MoS}_{2} @ A g N P s @ f l a t-S i$ substrate in the same way, to evaluate the role of PSi.

\subsection{Apparatus and characterization}

Surface morphologies of the MoS ${ }_{2} @ A g N P s @ P S i$ substrate were observed using scanning electron microscopy (SEM, Zeiss Gemini Ultra-55). The Raman spectroscopy was performed using a Raman spectrometer (Horiba HR Evolution 800) with laser excitation at $532 \mathrm{~nm}$. The crystalline quality and the singlecrystalline structure of $\mathrm{MoS}_{2}$ thin films were characterized by XRD (Bruker D8).

\section{Results and discussion}

As shown in Fig. 2(a), (c) and (e), after the texturing process, the samples shows clearly a typical surface morphology of PSi, which is composed of abundant well-separated pyramid arrays. The well-separated pyramid arrays can make contribution to the uniformity of the substrate. As illustrated in Fig. 2(a), well-ordered AgNPs are observed on the PSi substrate, which enables the AgNPs@PSi substrate to have high sensitivity and excellent uniformity. In order to observe more clearly, SEM image under a high magnification was obtained (the red circle in Fig. 2(a) is amplified), the distribution of the AgNPs is much homogeneous on both valley bottom and surface, as shown in Fig. 2(b). From the inset in Fig. 2(b), the average size of the AgNPs is about $35 \mathrm{~nm}$. Fig. 2(c) shows the SEM image of the $\mathrm{MoS}_{2} @ A g N P s @ P S i$ substrate, with the virtue of the direct growth method, the $\mathrm{MoS}_{2} @ A g N P s$ structure still maintains the well-ordered structure. The red circle in Fig. 2(c) is amplified in order to clearly observe, the size and 

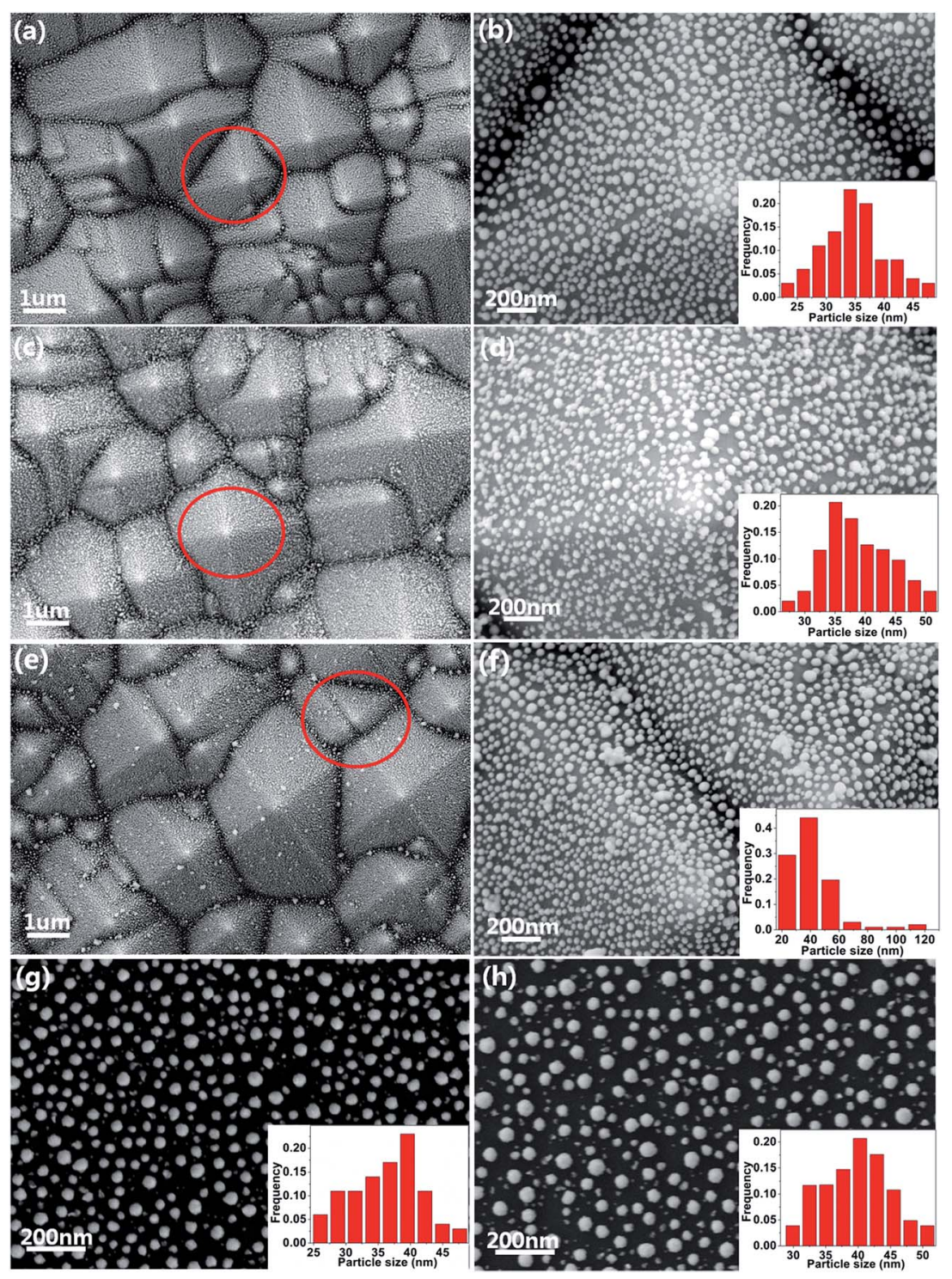

Fig. 2 (a) and (b) are SEM images of the AgNPs@PSi substrate in different magnification. (c) and (d) are SEM image of the MoS 2 (aAgNs@ $a$ PSi substrate in different magnification. (e) and ( $f$ ) are SEM images of the AgA aPSi substrate in different magnification. ( $g$ ) SEM image of the AgNPs@flat-Si substrate. (h) SEM image of the MoS 2 @AgNPs@flat-Si substrate.

interparticle distance of $\mathrm{MoS}_{2} @ A g N P s$ hybrid structure are similar to that of AgNPs in Fig. 2(b), as shown in Fig. 2(d). Fig. 2(e) and (f) are SEM images of the AgA@PSi substrate in different magnification. Obviously, there are many large size of the AgNPs in the valley bottom and surface of PSi. This phenomenon is attributed to the Ostwald ripening effect generated by the second annealing, ${ }^{36}$ which leads to the occurrence of AgNPs clusters. These particles will greatly weaken the Raman enhancement effect. But for the $\mathrm{MoS}_{2}$ @AgNPs@PSi substrate, the persistent high temperature did not make the $\mathrm{MoS}_{2} @ A g N P s$ hybrid structure produced clusters, probably because the AgNPs were wrapped up by the thin layer of $\mathrm{MoS}_{2}$, and the AgNPs can not fusion. SEM image of the AgNPs@flat-Si substrate is shown in Fig. 2(g). Similar with that of the AgNPs@PSi substrate, the AgNPs on the AgNPs@flat-Si substrate are also well-ordered. The $\mathrm{MoS}_{2}$ layers are also grown on the AgNPs@flat-Si forming the $\mathrm{MoS}_{2} @ A g N P s @ f l a t-S i$ substrate, as shown in Fig. 2(h).

In order to further investigate the distribution of the $\mathrm{MoS}_{2}$ layers, Raman spectra were obtained from the randomly selected twenty points on the $\mathrm{MoS}_{2} @ A g N P s @ P S i$ substrate, as shown in Fig. 3(a). The profile of Raman spectra of $\mathrm{MoS}_{2}$ from different positions is very similar, neither a shift in the characteristic Raman peaks nor a significant change in the Raman intensity occurs, indicating the uniformity of the $\mathrm{MoS}_{2}$ films in the whole substrate. Two Raman characteristic peaks of inplane vibrational mode $\mathrm{E}_{2 \mathrm{~g}}^{1}$ and out-of-plane vibrational mode $\mathrm{A}_{1 \mathrm{~g}}$ are all clearly seen. ${ }^{37}$ The full-width-half maximum (FWHM) values of $\mathrm{E}_{2 \mathrm{~g}}^{1}$ and $\mathrm{A}_{1 \mathrm{~g}}$ band respectively are 3-4 and 5-7 $\mathrm{cm}^{-1}$. The peak frequency difference $(\Delta)$ between $\mathrm{E}_{2 \mathrm{~g}}^{1}$ and $\mathrm{A}_{1 \mathrm{~g}}$ Raman modes can be used to identify the layer number of $\mathrm{MoS}_{2} \cdot{ }^{37}$ The value of $\Delta$ is obtained from the Raman spectra of $\mathrm{MoS}_{2}$ in 

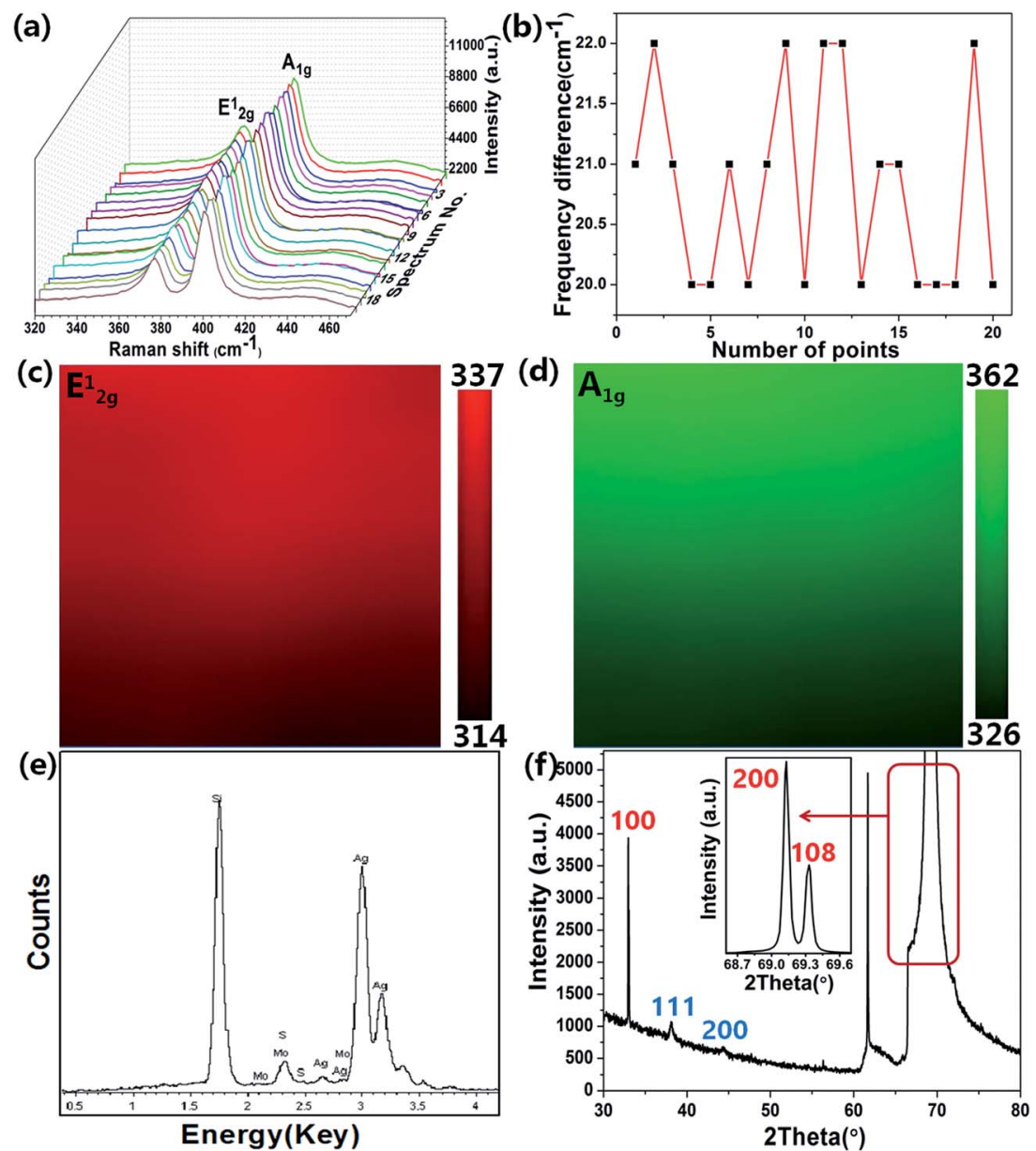

Fig. 3 (a) Raman spectra of the $M_{0} S_{2}$ layers from the $M_{0} S_{2} @ A g N P s @ P S i$ substrate. (b) The calculated peak frequency difference $(\Delta)$ between $E_{2 g}^{1}$ and $A_{1 g}$ Raman modes according to the Raman spectra in (a). (c) and (d) are Raman mapping of $E_{2 g}^{1}$ and $A_{1 g}$ peaks of MoS 2 , respectively. (e) EDS spectra of the $\mathrm{MoS}_{2}$ (aAgNPs@PSi substrate. (f) XRD pattern of the $\mathrm{MoS}_{2}$ (aAgNPs@PSi substrate.
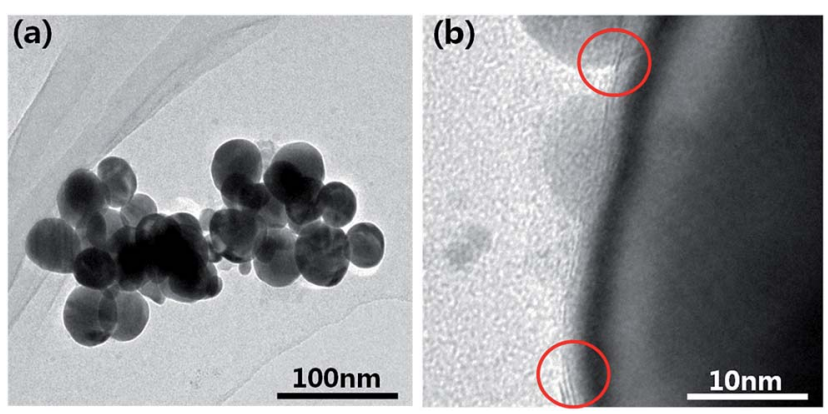

Fig. 4 (a) TEM image of the $M_{2} S_{2} @ A g N P s$ structure. (b) TEM image of the $\mathrm{MoS}_{2} @ A$ AgNPs structure in high magnification.

Fig. 3(a). As shown in Fig. 3(b), the values of $\Delta$ are in the range of 20-22 $\mathrm{cm}^{-1}$, which indicate that the as-grown $\mathrm{MoS}_{2}$ are 2-3 layers. In order to further demonstrate the uniformity of the $\mathrm{MoS}_{2}$ film, the $10 \times 10 \mu \mathrm{m}^{2}$ Raman mappings are obtained as shown in Fig. 3(c) and (d). The small color variations show favorable stability of the $\mathrm{E}_{2 \mathrm{~g}}^{1}$ and $\mathrm{A}_{1 \mathrm{~g}}$ Raman modes, indicating that the substrate is almost covered with $\mathrm{MoS}_{2}$ layers. Fig. 3(e) shows EDS spectra from the $\mathrm{MoS}_{2} @ A g N P s @ P S i$ substrate. The peaks associated with silicon, silver, sulfur and molybdenum are clearly observed. This shows that the $\mathrm{MoS}_{2} @$ @gNPs@PSi substrate is successfully prepared. Fig. 3(f) shows in-plane X-ray diffraction (XRD) pattern of the $\mathrm{MoS}_{2} @ A g N P s @ P S i$ substrate. The three strikingly peaks at $31.910,69.016$ and $69.158^{\circ}$ assigned as (100), (200) and (108) of the $\mathrm{MoS}_{2}$ crystals separately (powder diffraction fille (PDF) no. 751539). The diffraction peaks at 38.116 and 44.277 degree is attributed to the (111) and (200) crystal planes of AgNPs (powder diffraction fille (PDF) no. 04-0783). Based on the above mentioned images, we preliminary draw a conclusion that the $\mathrm{MoS}_{2}$ layers have successfully wrap the AgNPs and PSi.

Fig. 4(a) shows the TEM image of the $\mathrm{MoS}_{2}$ @AgNPs structure, we can see that the size of the $\mathrm{MoS}_{2} @ A g N P s$ structure is uniform. In order to clearly observe the $\mathrm{MoS}_{2}$ layers on the surface of the AgNPs, TEM image under a high magnification was obtained. As shown in Fig. 4(b), with the virtue of the direct growth method, the $\mathrm{MoS}_{2}$ layers tightly wrap the AgNPs, minimizing the loss of electromagnetic enhancement activity and endow the $\mathrm{MoS}_{2} @$ @gNPs@PSi substrate a high sensitivity. From the red circles in Fig. 4(b), the AgNPs were covered with two layer (above) or three layer (below) $\mathrm{MoS}_{2}$ can be clearly observed. 

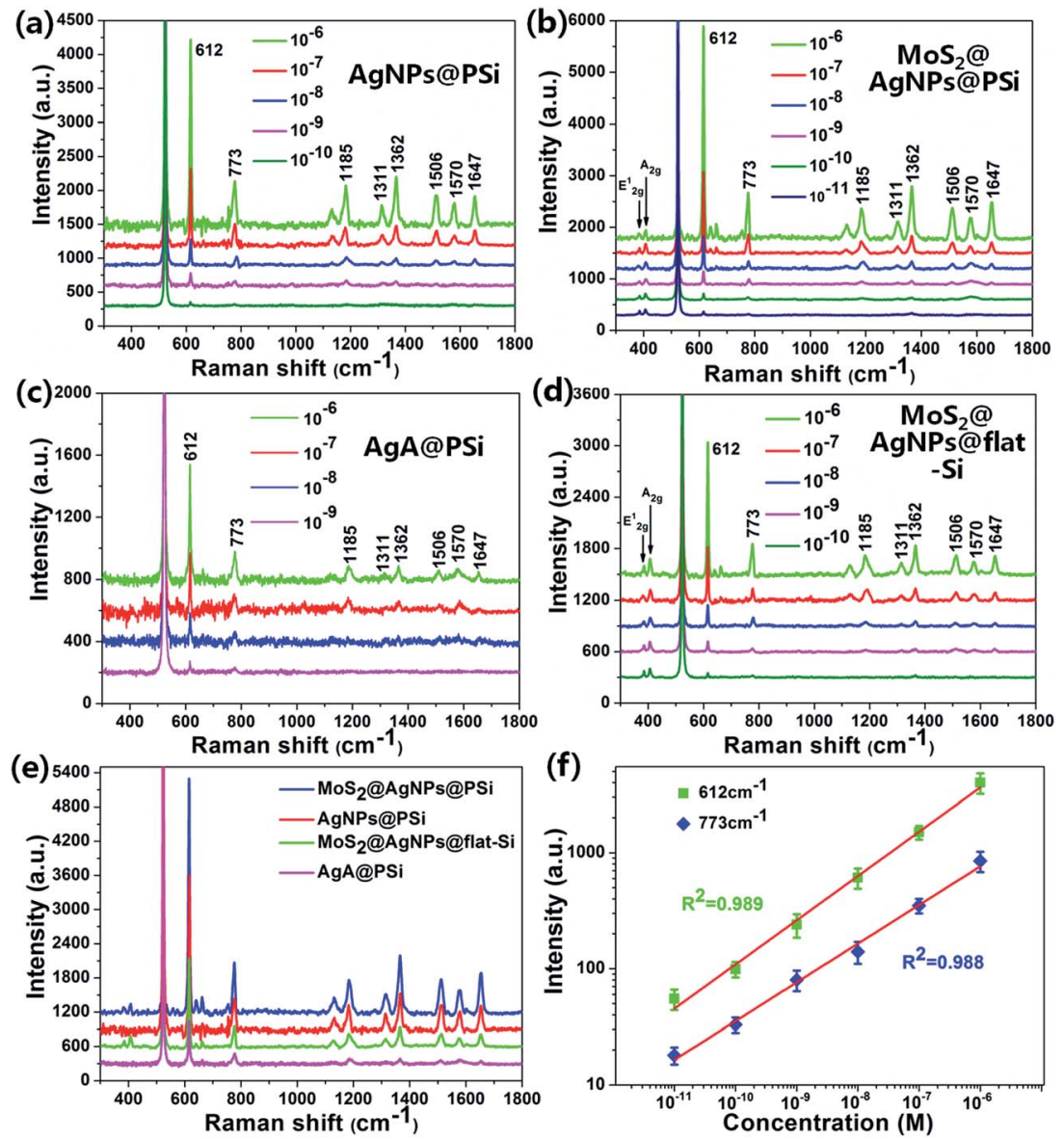

Fig. 5 (a) The Raman spectra of R6G on the AgNPs@PSi substrate from $10^{-6}$ to $10^{-10} \mathrm{M}$. (b) The Raman spectra of R6G on the MoS 2 (aAgNPs@PSi substrate from $10^{-6}$ to $10^{-11} \mathrm{M}$. (c) The Raman spectra of R6G on the AgA@PSi substrate from $10^{-6}$ to $10^{-9} \mathrm{M}$. (d) The Raman spectra of R6G on the $\mathrm{MoS}_{2} @ \mathrm{aAgNPs}$ aflat-Si substrate from $10^{-6}$ to $10^{-10} \mathrm{M}$. (e) The Raman spectra of R6G on the MoS 2 aAgNPs@PSi, AgNPs@PSi, MoS $(a-$ AgNPs@flat-Si and AgA@PSi substrates with concentration of $10^{-6} \mathrm{M}$. (f) Raman intensity of R6G at 612 and $773 \mathrm{~cm}^{-1}$ as a function of the molecular concentration on the $\mathrm{MoS}_{2} @ A$ AgNPs@PSi substrate, in log scale.

To estimate the SERS activity of the $\mathrm{MoS}_{2} @ A g N P s @ P S i$ substrate, we compared the behaviors of $\mathrm{MoS}_{2} @ A g N P s @ P S i$ substrate with that of AgNPs@PSi, AgA@PSi and $\mathrm{MoS}_{2} @$ AgNPs@flat-Si substrate. The R6G molecules with varied concentrations were chosen as the probe molecule and all the SERS spectra were implemented on the same conditions. The main Raman enhancement peaks of R6G are 612, 773, 1185, $1311,1362,1506,1570$ and $1647 \mathrm{~cm}^{-1}$. $^{38,39}$ The measured SERS intensity decay with the decrease of the R6G concentration for all prepared substrates. Through comparison of Fig. 5(a) and (b) we can see that the minimum detected concentration of R6G from the $\mathrm{MoS}_{2} @$ @AgNPs@PSi substrate is one order of magnitude lower than that from the AgNPs@PSi substrate, which can be as low as $10^{-11} \mathrm{M}$. The reason for this phenomenon is the $\mathrm{CM}$ enhancement effect from $\mathrm{MoS}_{2}$. Besides the $\mathrm{MoS}_{2}$ film also acts as the excellent adsorbent towards organic molecules. From Fig. 5(b) we can also clearly see the $\mathrm{E}_{2 \mathrm{~g}}^{1}$ and $\mathrm{A}_{1 \mathrm{~g}}$ two peaks, which proved the existence of $\mathrm{MoS}_{2}$. As shown in Fig. 5(c), the minimum detected concentration of R6G from the AgA@PSi substrate is only $10^{-9} \mathrm{M}$. Because of Ostwald ripening effect, the AgNPs with small diameter agglomerate into larger particles leads to the enhancement effect decreased. The minimum detected concentration of R6G from the $\mathrm{MoS}_{2} @ A g N P s @ P S i$ substrate is one order of magnitude lower than that from the $\mathrm{MoS}_{2} @$ AgNPs@flat-Si substrate, as shown in Fig. 5(d). The reason for this phenomenon is the well-separated pyramid arrays of PSi. Between the valley of PSi, the incident laser can produce effective oscillate. Futhermore, the scattering crosssection of the $\mathrm{MoS}_{2} @ A g N P s @ P S i$ substrate is relatively larger than the $\mathrm{MoS}_{2} @ A g N P s @ f l a t-S i$ system. All of these factors will lead to the enhancement of SERS signals. In order to be more aware of the Raman enhancement effect of all substrates, the Raman spectra of R6G on the $\mathrm{MoS}_{2} @ A g N P s @ P S i, A g N P s @ P S i$, $\mathrm{MoS}_{2} @ A g N P s @ f l a t-S i$ and AgA@PSi substrates with concentration of $10^{-6} \mathrm{M}$ are put in Fig. 5(e). From Fig. 5(e), we can clearly see that the SERS signal of the $\mathrm{MoS}_{2} @ A g N P s @ P S i$ substrate is the most powerful. The SERS enhancement factor (EF) for R6G were calculated according to the standard equation: ${ }^{40}$

$$
\mathrm{EF}=\frac{I_{\mathrm{SERS}} / N_{\mathrm{SERS}}}{I_{\mathrm{RS}} / N_{\mathrm{RS}}}
$$

where $I_{\mathrm{SERS}}$ and $I_{\mathrm{RS}}$ are respectively the intensity of the SERS spectra and the normal Raman spectra. $N_{\mathrm{SERS}}$ and $N_{\mathrm{RS}}$ are the 

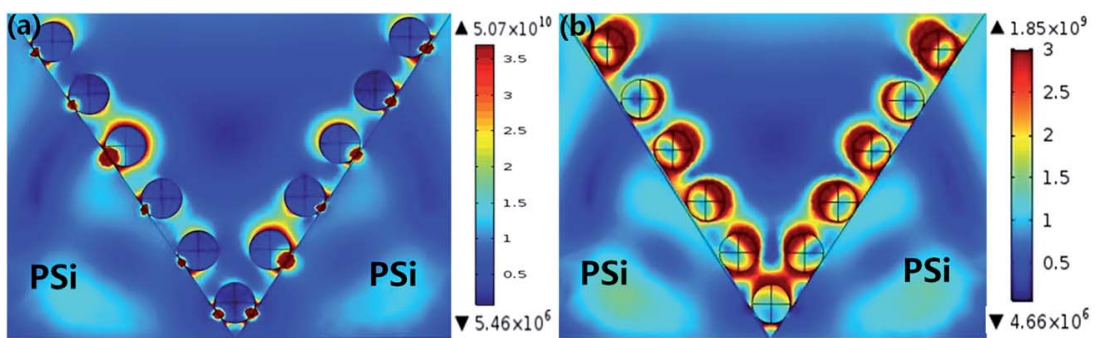

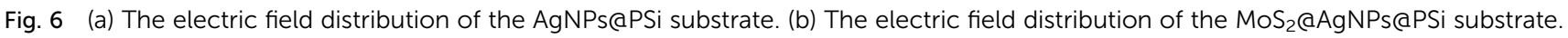

numbers of R6G molecules illuminated by the laser spot under SERS and normal Raman conditions, respectively. In order to calculate the $\mathrm{EF}$ of hybrid system, the equal volumes of R6G solution concentration of $10^{-4}, 10^{-10}, 10^{-11}, 10^{-9}$ and $10^{-10} \mathrm{M}$ were dispersed on the bare PSi, AgNPs@PSi, MoS ${ }_{2} @-$ AgNPs@PSi, AgA@PSi and $\mathrm{MoS}_{2} @ A g N P s @ f l a t-S i$, respectively. According to the above equation, the average $\mathrm{EF}$ is $9.55 \times 10^{6}$ for the $\mathrm{MoS}_{2} @ A g N P s @ P S i$ substrate. Follow the same method, the EF for AgNPs@PSi,AgA@PSi and MoS $_{2} @ A g N P s @ f l a t-S i$ is $7.88 \times 10^{5}, 1.02 \times 10^{5}$ and $7.27 \times 10^{5}$, respectively. These results clearly show that the $\mathrm{MoS}_{2} @ A g N P s @ P S i$ substrate have a excellent SERS effect. To further investigate the dynamic variation of Raman intensity versus the concentration of R6G on the $\mathrm{MoS}_{2} @ A g N P s @ P S i$ substrate, the linear fit curves are illustrated in Fig. 5(f). It is thoroughly indicate that reasonable linear response in log scale is achieved between the intensity of SERS signal and the R6G concentration. Based on the above mentioned results, we draw the conclusion that the $\mathrm{MoS}_{2}$ @AgNPs@PSi substrate can provide sensitive and reliable SERS signals.
With the purpose of further identify and better understand the SERS behaviors of the $\mathrm{MoS}_{2} @ A g N P s @ P S i$ substrate, we modeled the local electric field properties of the substrate by using commercial COMSOL software. We set the length and width of the PSi is $3 \mu \mathrm{m}$ and the height is $4 \mu \mathrm{m}$ according to the actual sample. Fig. 6(a) shown the electric field distribution of the AgNPs@PSi substrate that the diameter of AgNPs is $35 \mathrm{~nm}$ and the interparticle distance is $20 \mathrm{~nm}$. The $\mathrm{EM}$ for the substrate is calculated to be $1.06 \times 10^{3}$, and the $\mathrm{EF}$ calculated by this consequence is $1.26 \times 10^{12}$. The result of this simulation is higher than the experiment. There are two reasons for this phenomenon: first, the strong electric field mainly distributed in the place of AgNPs and PSi contact touching, but the probe molecule can not reach the place; second, the energy loss is also one of the important reasons. We also simulate and calculate the electric field of the $\mathrm{MoS}_{2} @$ @AgNPs@PSi substrate. As shown in Fig. 6(b), the diameter and interparticle distance of AgNPs are the same as that of the AgNPs@PSi substrate, the thickness of $\mathrm{MoS}_{2}$ is $1.3 \mathrm{~nm}$. According to the simulative results, the EM for $\mathrm{MoS}_{2} @ A g N P s @ P S i$ is $0.385 \times 10^{2}$ and the EF is $0.22 \times 10^{7}$. The

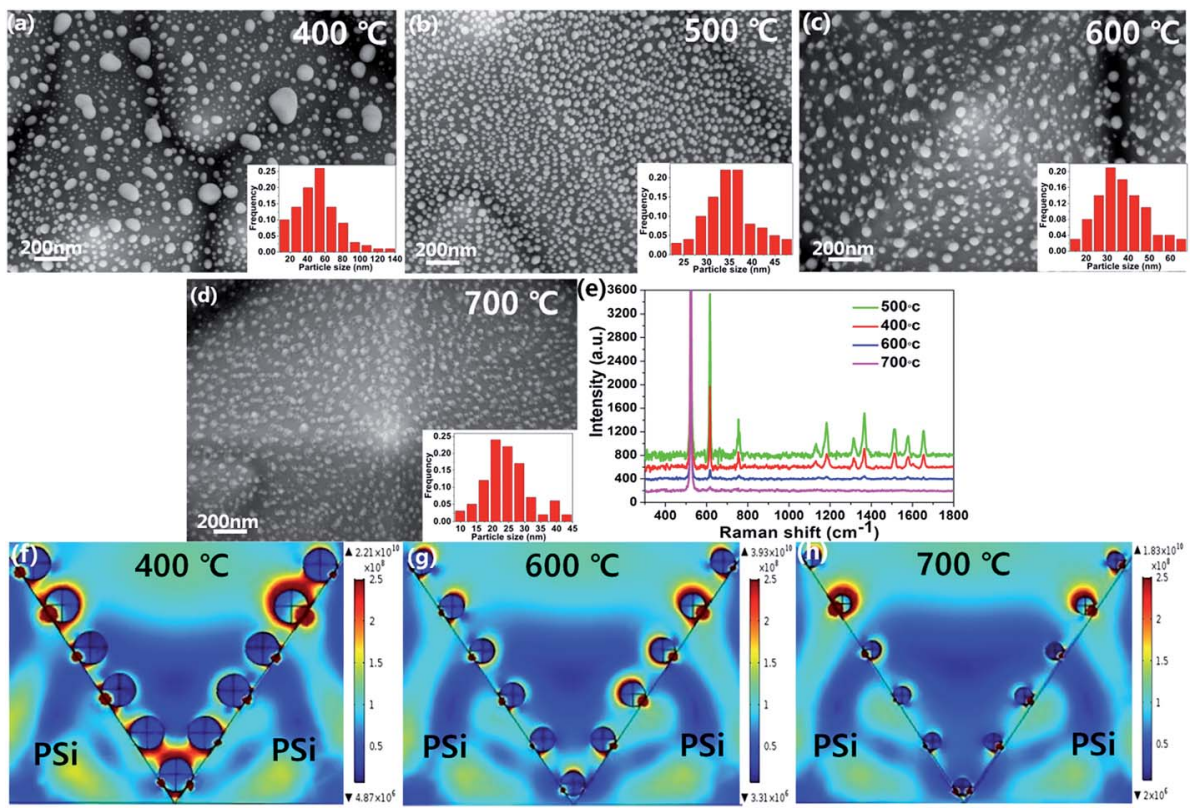

Fig. 7 (a), (b), (c) and (d) are SEM image and histograms of particle size distribution of the AgNPs for an annealing temperature at $400{ }^{\circ} \mathrm{C}$, $500{ }^{\circ} \mathrm{C}$, $600^{\circ} \mathrm{C}$ and $700^{\circ} \mathrm{C}$, respectively. (e) Raman spectra of R6G molecules that the concentration is $10^{-6} \mathrm{M}$ on the AgNPs@PSi substrate with different annealing temperature from $400{ }^{\circ} \mathrm{C}$ to $700^{\circ} \mathrm{C}$. (f), (g) and (h) are the electric field distribution of the AgNPs(aPSi substrate at $400{ }^{\circ} \mathrm{C}, 600{ }^{\circ} \mathrm{C}$ and $700{ }^{\circ} \mathrm{C}$, respectively. 
consequence is lower than the AgNPs@PSi, duo to the extra layer of $\mathrm{MoS}_{2}$ weakens the EM. But for the actual measurement, the CM provided by $\mathrm{MoS}_{2}$ is much larger than the EM weakened by $\mathrm{MoS}_{2}$. According to these theoretical results, a high sensitive SERS activity based on the $\mathrm{MoS}_{2} @ A g N P s @ P S i$ substrate can be accomplished.

In order to investigate the effect of different annealing temperatures on the AgNPs, we also prepared AgNPs@PSi at 400 ${ }^{\circ} \mathrm{C}, 600{ }^{\circ} \mathrm{C}$ and $700{ }^{\circ} \mathrm{C}$, respectively. As shown in Fig. 7(a), some of the larger particles are distributed in the surface and valleys of PSi for an annealing temperature at $400{ }^{\circ} \mathrm{C}$. The inset histograms shows the average size of AgNPs is about $47 \mathrm{~nm}$. As illustrated in Fig. 7(c), compare with the AgNPs@PSi substrate for an annealing temperature at $500{ }^{\circ} \mathrm{C}$, we can clearly see that the interparticle distance of AgNPs becomes larger and the density of AgNPs becomes smaller at $600{ }^{\circ} \mathrm{C}$. The reason for this phenomenon may be that the high temperature to speed up the mobility of silver molecules, a part of the silver molecules with argon left the PSi surface. From the inset in Fig. 7(c), the average size of AgNPs is about $34 \mathrm{~nm}$. When the temperature rises to 700 ${ }^{\circ} \mathrm{C}$, most of the silver is evaporated and the interparticle distance becomes much larger, as shown in Fig. 7(d). We can see some bubbles around the particles. Which may be the PSi can not resistant to high temperature resulting in a slight melting of the PSi. The average size of AgNPs is about $24 \mathrm{~nm}$ according to the inset in Fig. 7(d). Raman spectra of $10^{-6} \mathrm{M}$ R6G molecules on the AgNPs@PSi substrate for an annealing temperature at $400{ }^{\circ} \mathrm{C}, 500{ }^{\circ} \mathrm{C}, 600{ }^{\circ} \mathrm{C}$ and $700{ }^{\circ} \mathrm{C}$ was measured, respectively. As shown in Fig. $7(\mathrm{e})$, when the annealing temperature is $500{ }^{\circ} \mathrm{C}$, the Raman enhancement effect of the AgNPs@PSi substrate is the best. The electric field of the AgNPs@PSi substrate at $400{ }^{\circ} \mathrm{C}, 600{ }^{\circ} \mathrm{C}$ and $700{ }^{\circ} \mathrm{C}$ were also simulated. According to Fig. $7(\mathrm{f})-(\mathrm{h})$, the EF of annealing temperature at $400{ }^{\circ} \mathrm{C}, 600{ }^{\circ} \mathrm{C}$ and $700{ }^{\circ} \mathrm{C}$ calculated is $4.49 \times$ $10^{11}, 4.51 \times 10^{10}$ and $2.11 \times 10^{10}$, respectively. The results of these simulations also prove that the Raman enhancement effect of the AgNPs@PSi substrate is the best when the annealing temperature at $500{ }^{\circ} \mathrm{C}$.

In addition to the high enhancement activity of SERS signals from the $\mathrm{MoS}_{2} @ A g N P s @ P S i$ substrate, the hybrid system also shows many other excellent properties. Fig. 8(a) shows the SERS spectra of R6G molecules with concentration of $10^{-6} \mathrm{M}$ from 20
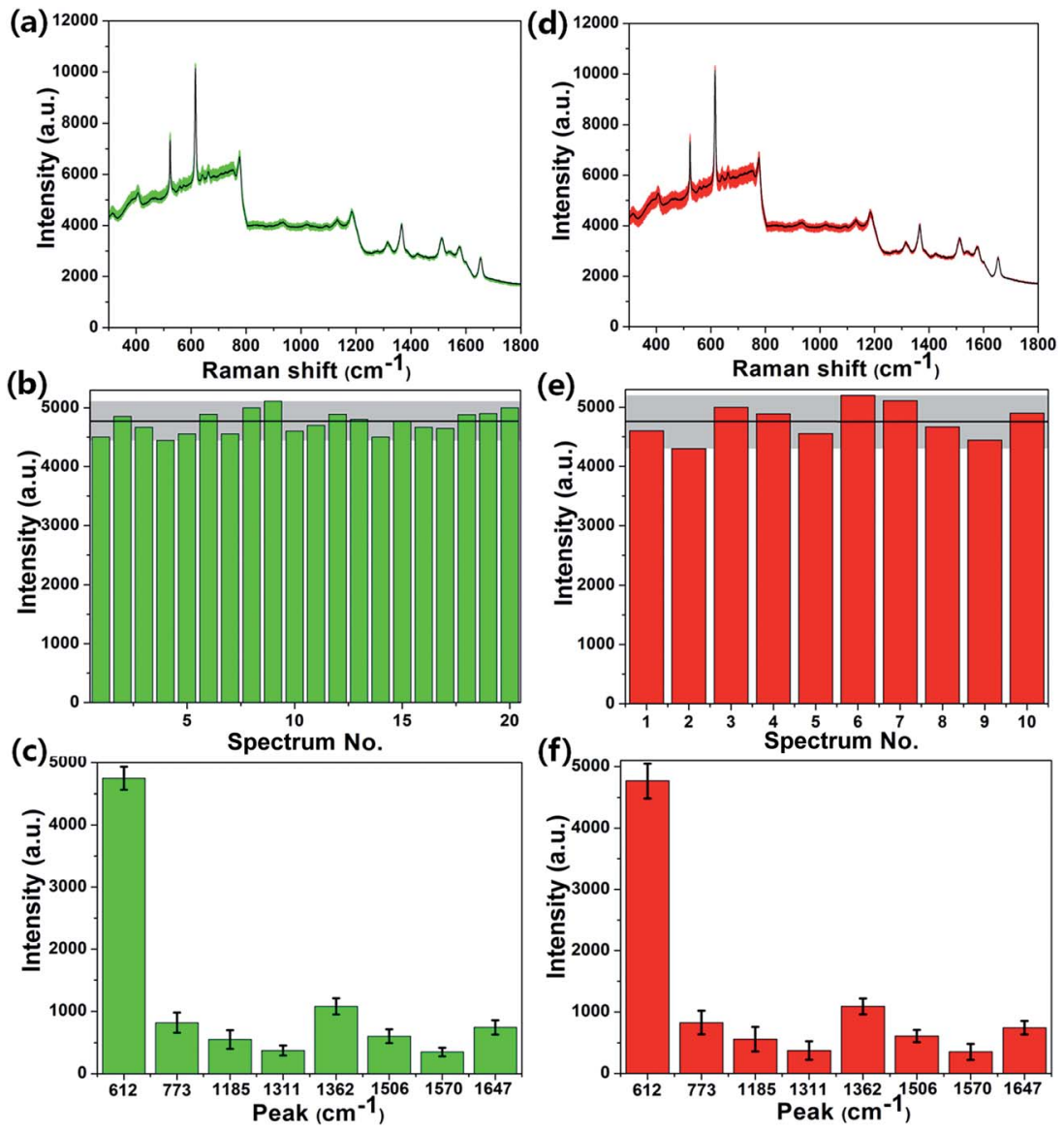

Fig. 8 (a) Average Raman spectra (black line) of the R6G molecules with concentration of $10^{-6} \mathrm{M}_{\text {from }} 20$ positions on a same MoS ${ }_{2}$ AgNPs@PSi substrate. (b) Intensity distribution for the peak at $612 \mathrm{~cm}^{-1}$ of the 20 Raman spectra. (c) Average intensity of characteristic peaks of the R6G molecules from the 20 Raman spectra. Error bars show the standard deviations. (d) Average Raman spectrum (black line) of the R6G molecules with concentration of $10^{-6} \mathrm{M}$ from $10 \mathrm{MoS}_{2} @$ AgNPs@PSi substrates in different batches. (e) Intensity distribution for the peak at 612 $\mathrm{cm}^{-1}$ of the 10 spectra from $10 \mathrm{MoS}_{2}$ (aAgNPs@PSi substrates in different batches. (f) Average intensity of characteristic peaks of the R6G molecules from the 10 spectra. Error bars show the standard deviations. 
positions on a same $\mathrm{MoS}_{2} @ A g N P s @ P S i$ substrate. These SERS spectrum overlap to form a green shaded area. The black curve in the green shaded area is the average spectrum of the 20 SERS spectra. The perfect overlap of Raman spectra from these positions leads to a quite narrow green shaded area, which indicates the excellent similarity of the 20 SERS spectra. Fig. 8(b) shows the intensity distribution for the peak at 612 $\mathrm{cm}^{-1}$ of the R6G molecules with $10^{-6} \mathrm{M}$ from 20 spectra on a same $\mathrm{MoS}_{2} @ A g N P s @ P S i$ substrate. The shadow area represents the vibration range of the peak at $612 \mathrm{~cm}^{-1}$. The black line in the shaded area is the average intensity. The maximum intensity deviation of the SERS spectra is given by the formula: ${ }^{41}$

$$
D=\frac{I-\bar{I}}{\bar{I}} \times 100 \%
$$

where $D$ is the maximum intensity deviation, $I$ is the maximum peak intensity and $\bar{I}$ is the average peak intensity of the SERS spectra. According to the formula, all the intensities of the peak at $612 \mathrm{~cm}^{-1}$ lie within a $7.6 \%$ variation range. Furthermore, the RSD of the peak at $612 \mathrm{~cm}^{-1}$ of the 20 spectra is $7.9 \%$, revealing the excellent uniformity of the $\mathrm{MoS}_{2} @ A g N P s @ P S i$ substrate. Average intensity of characteristic peaks of the R6G molecules from the 20 Raman spectra with error bar is shown in Fig. 8(c). Smaller standard deviations represent a lesser degree of dispersion, which also indicate the excellent uniformity of the $\mathrm{MoS}_{2} @ A g N P s @ P S i$ substrate. The excellent uniformity of the SERS signals from the $\mathrm{MoS}_{2} @ A g N P s @ P S i$ substrate can be attributed to at least three aspects: first, the homogeneous AgNPs formed by annealing is the most important reason for the uniformity of the substrate; second, the well-separated pyramid arrays of PSi can make contribution to the uniformity of substrate; third, the $\mathrm{MoS}_{2}$ layer reduces the additional signal by isolating the metal nanoparticles from the R6G molecules and enables the SERS analysis with more well defined molecular interactions. We use the same approach to evaluate the reproducibility of the $\mathrm{MoS}_{2} @ A g N P s @ P S i$ substrate. We measured SERS spectra of the R6G molecules with concentration of $10^{-6}$ M from $10 \mathrm{MoS}_{2} @ A g N P s @ P S i$ substrates in different batches. As shown in Fig. 8(d), the narrow red shaded area indicates that the $\mathrm{MoS}_{2} @ A g N P s @ P S i$ substrate has a good reproducibility. According to the formula (2), the height of all columns in Fig. $8(\mathrm{e})$ is within the range of $9 \%$. The RSD of the peak at 612 $\mathrm{cm}^{-1}$ of the 10 SERS spectra from different $\mathrm{MoS}_{2} @ A g N P s @ P S i$ is $9.3 \%$. Besides, the characteristic peaks of the R6G molecules from the 10 spectra has a smaller degree of dispersion, as shown in Fig. 8(f). These results fully demonstrate that the SERS signal of the $\mathrm{MoS}_{2} @ A g N P s @ P S i$ substrate is reproducible.

As shown in Fig. 9(a), original SERS spectra of R6G molecules that the concentration is $10^{-6} \mathrm{M}$ were collected from five randomly selected spots on the AgNPs@PSi substrate and the $\mathrm{MoS}_{2} @ A g N P s @ P S i$ substrate, respectively. Obviously, background fluorescence of the $\mathrm{MoS}_{2} @ A g N P s @ P S i$ substrate was significantly decreased compare with the AgNPs@PSi substrate, this phenomenon can be explained by the fluorescence quenching effect of $\mathrm{MoS}_{2}$. The characteristic of $\mathrm{MoS}_{2}$ can contribute to the uniformity of SERS signals from the $\mathrm{MoS}_{2}$ @AgNPs@PSi substrate. In order to further investigate the stability of the $\mathrm{MoS}_{2} @ A g N P s @ P S i$ substrate, the R6G concentration of $10^{-6} \mathrm{M}$ was selected to compare the stability of the AgNPs@PSi and the $\mathrm{MoS}_{2} @ A g N P s @ P S i$ substrates. The Raman intensity was measured every five days under the room temperature. As shown in Fig. 9(b), the measured SERS intensity attenuation of the AgNPs@PSi substrate is very obvious with the increase of the number of days. But for the $\mathrm{MoS}_{2} @ A g N P s @ P S i$ substrate, the attenuation of the Raman intensity is very weak, as shown in Fig. 9(c). We made a line chart to make the contrast
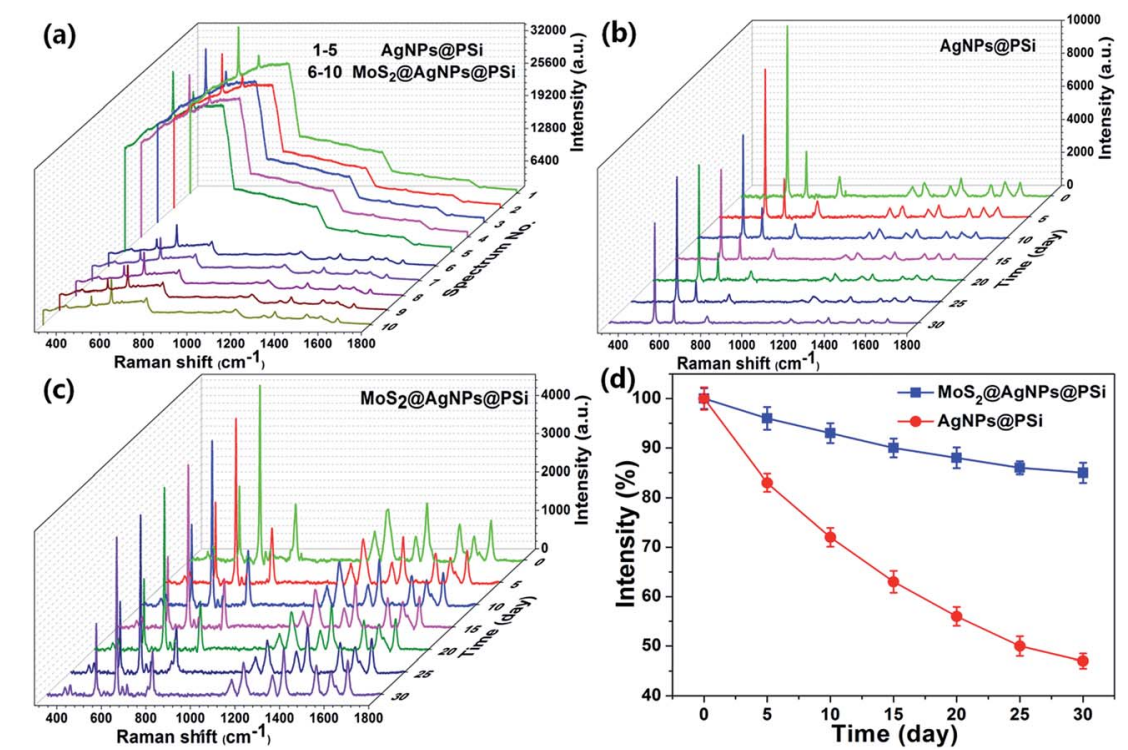

Fig. 9 (a) Original Raman spectra of R6G molecules that the concentration is $10^{-6} \mathrm{M}$ were collected from the AgNPs@PSi and MoS 2 @AgNPs@PSi substrates. (b) The Raman spectra of the AgNPs@PSi substrate was measured every five days under the room temperature. (c) The Raman spectra of the $\mathrm{MoS}_{2}$ @AgNPs@PSi substrate was measured every five days under the room temperature. (d) The Raman intensity changes with time obtained from the AgNPs@PSi and MoS 2 aAgNPs@PSi substrates. 
even more clear (Fig. 9(d)). One month later, the average intensity of peak at $612 \mathrm{~cm}^{-1}$ obtained from the AgNPs@PSi substrate is decreased 53\%. Uplifting, the average intensity of the $\mathrm{MoS}_{2} @ A g N P s @ P S i$ substrate is dropped only 15\%. Through this comparison we draw a preliminary conclusion that the $\mathrm{MoS}_{2}$ film can tightly wrap the AgNPs and isolate it from the outside environment, effectively protect AgNPs from oxidation and endow the $\mathrm{MoS}_{2} @ A g N P s @ P S i$ substrate long-term stability.

\section{Conclusions}

In conclusion, by combining AgNPs, PSi and $\mathrm{MoS}_{2}$, a new SERS substrate has been presented based on $\mathrm{MoS}_{2} @ A g N P s @ P S i$.We using R6G as probe molecule, the SERS results indicate that the $\mathrm{MoS}_{2} @ A g N P s @ P S i$ substrate has excellent sensitivity, uniformity, reproducibility and stability. The SERS behaviors of the AgNPs@PSi and $\mathrm{MoS}_{2} @ A g N P s @ P S i$ substrates obtained in experiment were confirmed by theoretical calculations with the assist of a commercial COMSOL software. These experimental and theoretical results imply that the $\mathrm{MoS}_{2} @ A g N P s @ P S i$ is expected to be an effective substrate for label-free SERS detections.

\section{Acknowledgements}

The authors are grateful for financial support from the National Natural Science Foundation of China (11674199, 11604040, 11474187 and 11404193), Shandong Province Natural Science Foundation (ZR2014FQ032 and ZR2013HQ064), Excellent Young Scholars Research Fund of Shandong Normal University.

\section{References}

1 A. Barhoumi and J. N. Halas, J. Am. Chem. Soc., 2010, 132, 12792-12793.

2 K. Lee, V. P. Drachev and J. Irudayaraj, ACS Nano, 2011, 5, 2109-2117.

3 G. B. Jung, Y. M. Bae, Y. J. Lee, S. H. Ryu and H. K. Park, Appl. Surf. Sci., 2013, 282, 161-164.

4 X. M. Qian and S. M. Nie, Chem. Soc. Rev., 2008, 37, 912-920.

5 J. Jiang, K. Bosnick, M. Maillard and L. Brus, J. Phys. Chem. B, 2003, 107, 9964-9972.

6 W. Li, P. H. C. Camargo, X. Lu and Y. Xia, Nano Lett., 2009, 9, 485-490.

7 D. Graham, D. G. Thompson, W. E. Smith and K. Faulds, Nat. Nanotechnol., 2008, 3, 548-551.

8 S. J. Lee, A. R. Morrill and M. Moskovits, J. Am. Chem. Soc., 2006, 128, 2200-2201.

9 X. Liu, J. Wang, Y. Wu, T. Fan, Y. Xu, L. Tang and Y. Ying, Sci. Rep., 2015, 5, 17167.

10 R. A. Tripp, R. A. Dluhy and Y. Zhao, Nanotoday, 2008, 3, 3137.

11 Y. Sun, K. Liu, X. Hong, M. Chen, J. Kim, S. Shi, J. Wu, A. Zett and F. Wang, Nano Lett., 2014, 14, 5329-5334.

12 D. Zhang, Y. C. Wu, M. Yang, X. Liu, C. Ó. Coileáin, H. Xu, M. Abid, M. Abid, J. J. Wang, I. V. Shvets, H. Liu, Z. Wang,
H. Yin, H. Liu, B. S. Chun, X. Zhang and H. C. Wu, RSC Adv., 2016, 6, 99053-99059.

13 D. Zhang, Y. C. Wu, M. Yang, X. Liu, C. Ó. Coileáin, M. Abid, M. Abid, J. J. Wang, I. Shvets, H. Xu, B. S. Chun, H. Liu and H. C. Wu, Sci. Rep., 2016, 6, 30320.

14 Y. Deng, M. Chen, J. Zhang, Z. Wang, W. Huang, Y. Zhao, J. P. Nshimiyimana, X. Hu, X. Chi, G. Hou, X. Zhang, Y. Guo and L. Sun, Nano Res., 2016, 9, 1682-1688.

15 S. Su, H. Sun, W. Cao, J. Chao, H. Peng, X. Zuo, L. Yuwen, C. Fan and L. Wang, ACS Appl. Mater. Interfaces, 2016, 8, 6826-6833.

16 J. Zhao, Z. Zhang, S. Yang, H. Zheng and Y. Li, J. Alloys Compd., 2013, 559, 87-91.

17 S. S. Singha, D. Nandi and A. Singha, $R S C A d v$., 2015, 5, 24188-24193.

18 Z. Li, S. Jiang, Y. Huo, M. Liu, C. Yang, C. Zhang, X. Liu, Y. Sheng, C. Li and B. Man, Opt. Express, 2016, 24, 2609726108.

19 P. X. Chen, H. W. Qiu, S. C. Xu, X. Y. Liu, Z. Li, L. T. Hu, C. H. Li, J. Guo, S. Z. Jiang and Y. Y. Huo, Appl. Surf. Sci., 2016, 375, 207-214.

20 S. Xu, B. Man, S. Jiang, J. Wang, J. Wei, S. Xu, H. Liu, S. Gao, H. Liu, Z. Li, H. Li and H. Qiu, ACS Appl. Mater. Interfaces, 2015, 7, 10977-10987.

21 C. Zhang, M. Liu, B. Y. Man, S. Z. Jiang, C. Yang, C. S. Chen, D. J. Feng, D. Bi, F. Y. Liu, H. W. Qiu and J. X. Zhang, CrystEngComm, 2014, 16, 8941-8945.

22 H. W. Qiu, S. C. Xu, P. X. Chen, S. S. Gao, Z. Li, C. Zhang, S. Z. Jiang, M. Liu, H. S. Li and D. J. Feng, Appl. Surf. Sci., 2015, 332, 614-619.

23 C. Zhang, B. Y. Man, C. Yang, S. Z. Jiang, M. Liu, C. S. Chen, S. C. Xu, X. G. Gao and Z. C. Sun, Sci. Adv. Mater., 2014, 6, 399-404.

24 S. Xu, S. Jiang, J. Wang, J. Wei, W. Yue and Y. Ma, Sens. Actuators, B, 2016, 222, 1175-1183.

25 F. Giorgis, E. Descrovi, A. Chiodoni, E. Froner, M. Scarpa, A. Venturello and F. Geobaldo, Appl. Surf. Sci., 2008, 254, 7494-7497.

26 A. Chiolerio, A. Virga, P. Pandolfi, P. Martino, P. Rivolo, F. Geobaldo and F. Giorgis, Nanoscale Res. Lett., 2012, 7, 1-7.

27 Y. Q. Wang, S. Ma, Q. Q. Yang and X. J. Li, Appl. Surf. Sci., 2012, 258, 5881-5885.

28 B. Kiraly, S. Yang and T. J. Huang, Nanotechnology, 2013, 24, 245704.

29 L. M. Chen and Y. N. Liu, CrystEngComm, 2011, 13, 64816487.

30 J. Yang, J. B. Li, Q. H. Gong, J. H. Teng and M. H. Hong, Nanotechnology, 2014, 25, 465707.

31 K. Q. Peng, Y. J. Yan, S. P. Gao and J. Zhu, Adv. Mater., 2002, 14, 1164.

32 X. Sun, N. Wang and H. Li, Appl. Surf. Sci., 2013, 284, 549-555.

33 G. Seniutinas, G. Gervinskas, R. Verma, B. D. Gupta, F. Lapierre, P. R. Stoddart, F. Clark, S. L. McArthur and S. Juodkazis, Opt. Express, 2015, 23, 6763-6772.

34 C. Zhang, B. Y. Man, S. Z. Jiang, C. Yang, M. Liu, C. S. Chen, S. C. Xu, H. W. Qiu and Z. Li, Appl. Surf. Sci., 2015, 347, 668672. 
35 C. Zhang, S. Z. Jiang, Y. Y. Huo, A. H. Liu, S. C. Xu, X. Y. Liu, Z. C. Sun, Y. Y. Xu, Z. Li and B. Y. Man, Opt. Express, 2015, 23, 24811-24821.

36 G. Madras and B. J. Mccoy, J. Chem. Phys., 2003, 119, 16831693.

37 C. Lee, H. Yan, L. E. Brus, T. F. Heinz, J. Hone and S. Ryu, ACS Nano, 2010, 4, 2695-2700.
38 Y. Chen, G. Tian, K. Pan, C. Tian, J. Zhou, W. Zhou, Z. Ren and H. Fu, Dalton Trans., 2012, 41, 1020-1026.

39 Y. K. Kim, S. W. Han and D. H. Min, ACS Appl. Mater. Interfaces, 2012, 4, 6545-6551.

40 S. Xu, J. Wang, Y. Zou, H. Liu, G. Wang, X. Zhang, S. Jiang, Z. Li, D. Cao and R. Tang, RSC Adv., 2015, 5, 90457-90465.

41 S. Xu, S. Jiang, G. Hu, J. Wei, L. Wang, J. Zhang and Q. Li, Laser Phys., 2015, 25, 115601. 